\title{
SPDT RF MEMS Switch Using A Single Bias Voltage And Based On Dual Series And Shunt Capacitive MEMS Switches
}

\author{
T. Ketterl ${ }^{1}$ and T. Weller ${ }^{2}$ \\ ${ }^{1}$ University of South Florida, Center for Ocean Technology, $1407^{\text {th }}$ Ave. S., St. Petersburg, FL 33701, USA, \\ (727) 553-1288 \\ ${ }^{2}$ University of South Florida, Department of Electrical Engineering, 4202 E. Fowler Ave., Tampa, FL 33613, USA
} (813) 974-4851

\begin{abstract}
A coplanar waveguide (CPW) single-pole double-throw (SPDT) X-band RF MEMS switch that can be actuated between states by applying a single voltage is introduced. Since a signal can be transmitted to one of the output ports without biasing, this has the potential to reduce the complexity of the required biasing network. The switch consists of a series and a shunt capacitive MEMS switch separated by a quarter wavelength transmission line. The shunt switch section was fabricated and measured separately and shown to have an insertion loss of $0.25 \mathrm{~dB}$ and isolation of $33 \mathrm{~dB}$ at $10 \mathrm{GHz}$. A SPDT 3-port switch was fabricated and port isolations of about $15 \mathrm{~dB}$ and an insertion loss of $1 \mathrm{~dB}$ were obtained in the up-state. In the down-sate, $40 \mathrm{~dB}$ of isolation with a $1 \mathrm{~dB}$ insertion loss were measured. The actuation voltage was $35 \mathrm{~V}$.
\end{abstract}

\section{INTRODUCTION}

Single-pole double-throw (SPDT) switches are an integral part of many microwave systems such as transceivers, where they are used to switch a device between receiver and transmitter modes, and receiver front ends for diversity application. It has already been shown that RF SPDT switches utilizing micro-electromechanical systems (MEMS) technology can be an attractive alternative to their solid state counterparts due to their low insertion loss, high isolation and low power consumption at microwave frequencies [1-5]. These types of MEMS switches utilize electrostatic actuation to change the signal path and generally require two independent voltages to switch between the two ports. This means that for the switch to operate, a bias voltage would need to be applied constantly and could require complex biasing systems if these switches were to be used in larger switching networks, such as phased array networks.

In this paper, a RF MEMS SPDT switch with a coplanar waveguide (CPW) topology operating in the Xband is introduced that has the advantage of needing just a single voltage to route the signal between the two output ports, i.e. with no applied bias, the signal flows through one port but gets routed through the other port when a bias is applied. The advantage of this is that the switch only needs to be powered in one state therefore simplifying the biasing as well as potentially increasing the switching lifetime of the device.

\section{SWITCH OPERATION}

The demonstrated MEMS switch actuation mechanism is based on electrostatically actuated fixed-fixed metal bridges suspended over the center conductors of CPW transmission lines. An air gap separates the bridge from the center conductor when no bias is applied. When a voltage is applied between these two metals, the electrostatic force starts to pull the bridge towards the lower conductor. The bias is then increased until a threshold voltage is reached at which point the bridge's spring force is overcome and the bridge snaps down and makes contact with the bottom signal line. For capacitive switches, a dielectric layer is usually placed on top of the bottom metal to provide DC isolation.

In the case of the SPDT switch presented in this paper, the difference in potential when a voltage is applied actuates two separate MEMS capacitive switches simultaneously; one in series configuration and the other in shunt configuration, as shown in Fig. 1. When both switches are in the upstate, the signal flows through port 2 and is isolated from port 3 , due to the air gap in the series switch. When a bias is applied to the device, causing both switches to actuate simultaneously, the signal will now flow through port 3 due to capacitive coupling in the down state of the series switch. The actuated shunt switch shorts the signal at port 2, which is transformed to an RF open due to the quarter wavelength separation from the series switch. This provides the isolation from port 2 in the down state. Since the materials and dimensions of the dielectric and actuation structures are identical for both switches, near simultaneous actuation can be accomplished with a single voltage. As already mentioned, previously demonstrated SPDT MEMS switches that have also utilized a dual switch design with quarter wavelength sections need two separate biasing circuits to actuate each device alternatively. In the design presented here, no switching voltage supply is needed since the switching of the signal between ports is controlled by a single bias supply. 


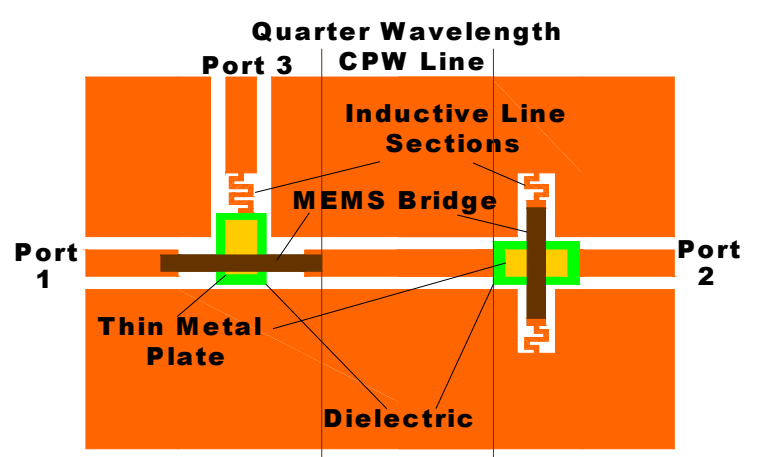

Fig. 1. SPDT switch layout (switch length not drawn to scale). The CPW line consists of $100 \mu \mathrm{m}$ wide center conductors and $50 \mu \mathrm{m}$ wide slots. The thin metal plates measure 100x200 $\mu \mathrm{m}$ and the actuation beams are $415 \mu \mathrm{m}$ long and $60 \mu \mathrm{m}$ wide. The overall dimension of the SPDT switch is $1.4 \times 3.42 \mathrm{~mm}$. Inductive line sections were used for resonant tuning in the down-state.

\section{FABRICATION PROCESS}

The shunt switch section was fabricated separately for initial testing purposes. The center conductor directly underneath the actuation area is slightly thinner $(0.2 \mu \mathrm{m})$ than the rest of the $\mathrm{CPW}$ line thickness $(0.8 \mu \mathrm{m})$. This was done to improve the planarity of the shunt beams. After a $0.25 \mu \mathrm{m}$ layer of PECVD nitride layer (for DC isolation) was deposited, patterned and etched, a thin metal plate was fabricated on top of the dielectric to help offset the degradation in the on-state capacitance which can occur due to warping of the shunt beams. The actuation beams were gold electroplated with the rest of the CPW lines to a thickness of $0.8 \mu \mathrm{m}$ on top of a 1.8 $\mu \mathrm{m}$ thick polymethyl methacrylate (PMMA) sacrificial layer. The devices were then released using a critical point drying technique. The substrate used was $400 \mu \mathrm{m}$ thick high resistivity $<100>$ silicon with a $1 \mu \mathrm{m}$ thick silicon dioxide layer on both sides. The SPDT switches were fabricated using the same process, except that the PMMA layer was about $1.5 \mu \mathrm{m}$ thick. Air bridges were also wire bonded over the series switch sections to equalize the ground planes of the SPDT witch. Fig. 2 shows an image of a fabricated switch. The fabrication process is described in detail in Fig. 3.
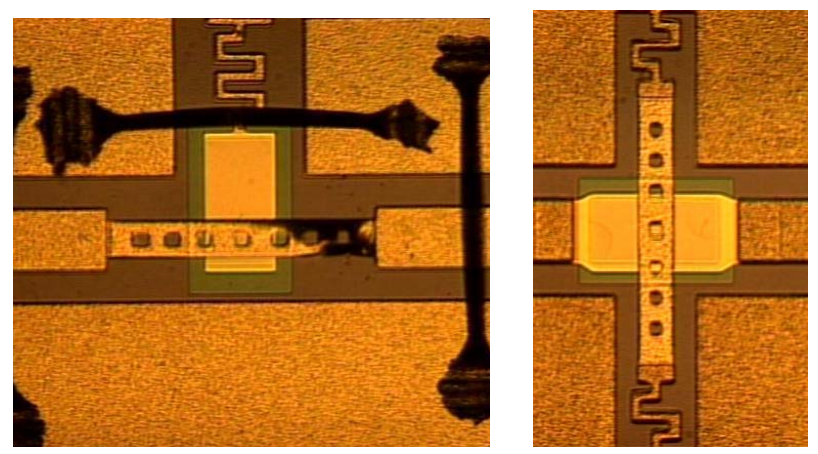

Fig. 2. Optical image of a fabricated SPDT switch. The series section is shown on the left, the shunt section on the right.

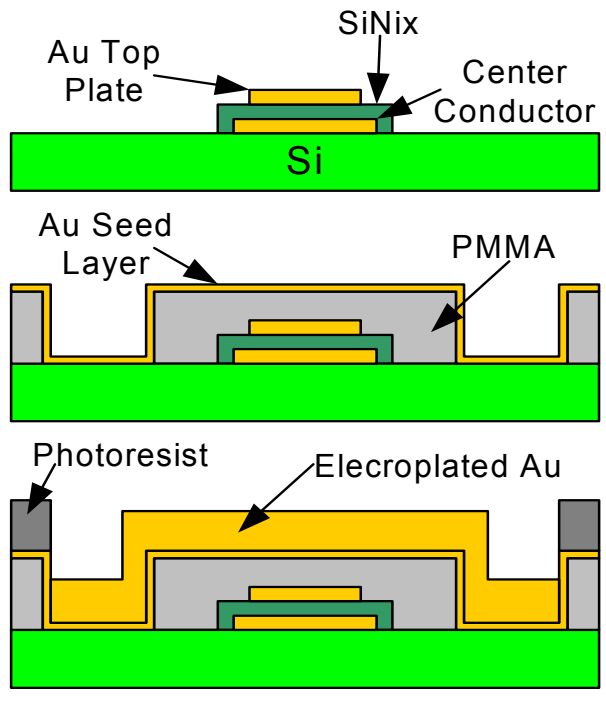

(a)
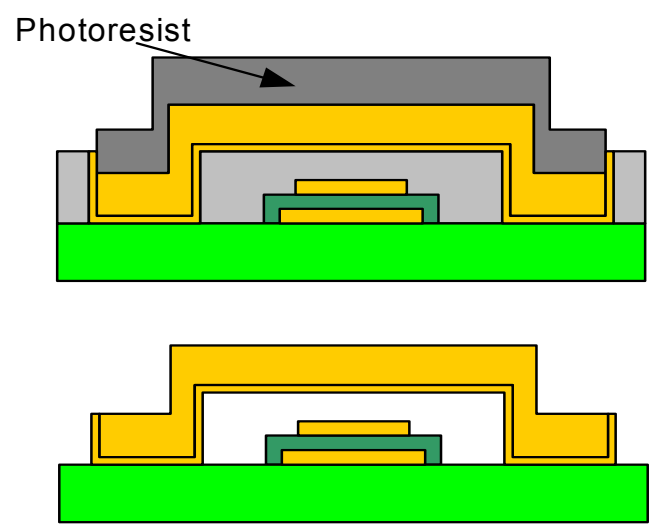

(e)

Fig. 3. Fabrication process flow: (a) a $0.2 \mu \mathrm{m}$ thick $\mathrm{Cr} / \mathrm{Au}$ center conductor section is thermally evaporated using lift-off. $0.25 \mu \mathrm{m}$ thick PECVD nitride is deposited and etched back after which a thermally evaporated $0.1 \mu \mathrm{m}$ thick $\mathrm{Cr} / \mathrm{Au}$ layer is placed on top of the nitride. (b) PMMA is spun on to a thickness of about $1.5-1.8 \mu \mathrm{m}$. A thin $\mathrm{Ti} / \mathrm{Au}$ seed layer is thermally evaporated to coat the sample. Photoresist is then spun on and patterned to mask the PMMA for the CPW and bridge plating process. (c) The CPW lines and the bridges are gold electroplated to about $0.8 \mu \mathrm{m}$ thickness. (d) Photoresist is patterned to protect the CPW and bridge layer when the Ti/Au seed layer is removed through wet etching. (e) The PMMA is dissolved using resist stripper and finally released using a critical point drying technique.

\section{RESULTS}

Fig. 4 shows a comparison of the simulated and measured performance from 1 to $16 \mathrm{GHz}$ of the individual shunt switch. Simulations were performed using Agilent ADS 2003A (6) and measurements were made using a Wiltron 360 vector network analyzer and Karl-Suss probe station with GGB $150 \mu \mathrm{m}$ pitch probes. A TRL calibration was performed initially. The bias was applied through the RF ports using high voltage bias tees. The switch actuated at $40 \mathrm{~V}$. An isolation of $>30 \mathrm{GHz}$ at $10 \mathrm{GHz}$ was achieved at resonance in the down state. In the up state, an insertion loss of 0.25 was measured, also at $10 \mathrm{GHz}$. 


\section{MEMS Shunt Switch Response}

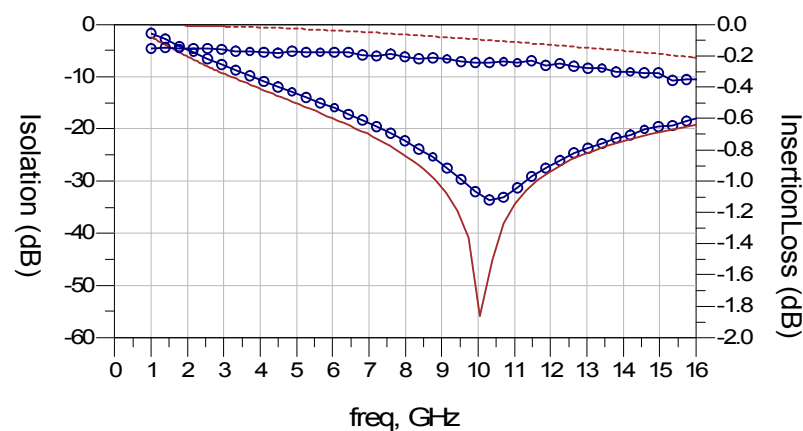

Fig. 4. Measured (lines with markers) and simulated (solid lines) data comparison of a shunt MEMS switch. Isolation and insertion loss are shown.

The SPDT switch was measured using an Anritsu 37397C vector network analyzer and 4-port switching box. The VNA and the 4-port box were controlled using the NISTCAL multi-port calibration and measurement software. The port convention shown in Fig. 1 was used during the measurements and for the simulations. With no applied bias, i.e. with the MEMS bridges in the upstate and the signal getting transmitted between ports 1 and 2 , a port 1 to port 3 isolation of $15 \mathrm{~dB}$ was measured. This was about the same for the isolation between ports 2 and 3. An insertion loss of $1 \mathrm{~dB}$ was measured between ports 1 and 2. Fig. 5 shows a comparison between the measured and simulated results. It can be observed that the isolation was about $5 \mathrm{~dB}$ lower for the measured switches since the air gap of the MEMS bridges was lower then the specified gap of $1.8 \mu \mathrm{m}$ of the simulated switches . Fig. 6 shows a comparison of the return losses at each port between the measured and simulated data.

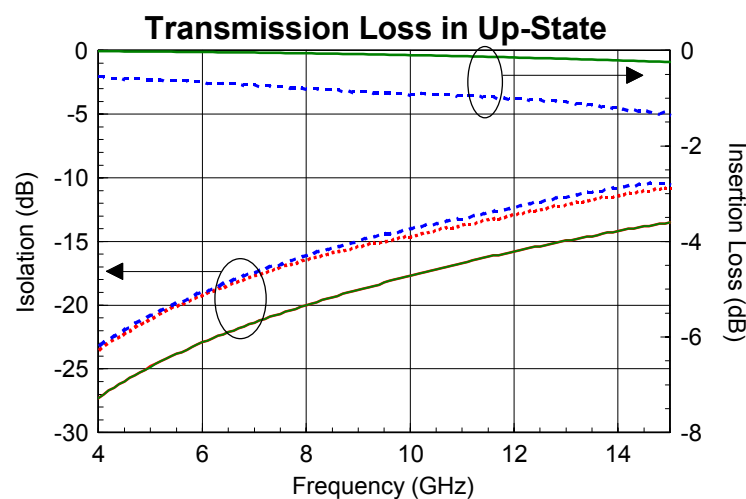

Fig. 5. Port 3 to port 1 (red dotted line) and port 2 to port 3 (blue dashed line) isolation are compared to simulated results with the switch in the up (unactuated) state. Measured and simulated port 2 to port 1 insertion loss (blue dashed line) is also shown. Green solid lines are simulated data.

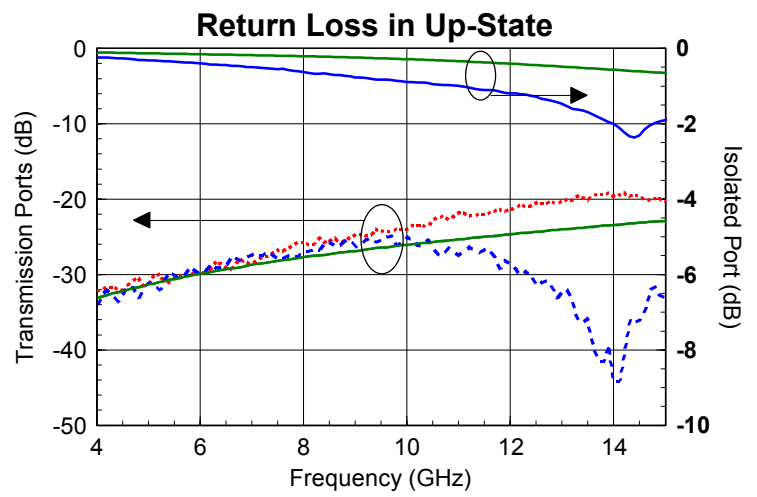

Fig. 6. Port 1 (red dotted line) and port 2 (blue dashed line) return losses are shown and compared to simulated results in the up-state. Return loss of the isolated Port 3 (solid blue line) is also plotted. Green solid lines are simulated data.

The switches were then actuated with a $35 \mathrm{~V}$ bias. The isolation and insertion loss response of the switch are shown in Fig. 7. The port 2 to port 1 and port 3 isolation reached nearly $40 \mathrm{~dB}$ in the down-state. Again, an insertion loss of about $1 \mathrm{~dB}$ was measured from port 1 to port 3. The return loss at each port in the down-state is shown in Fig. 8. Both plots again show a comparison of the measured data to corresponding simulated results.

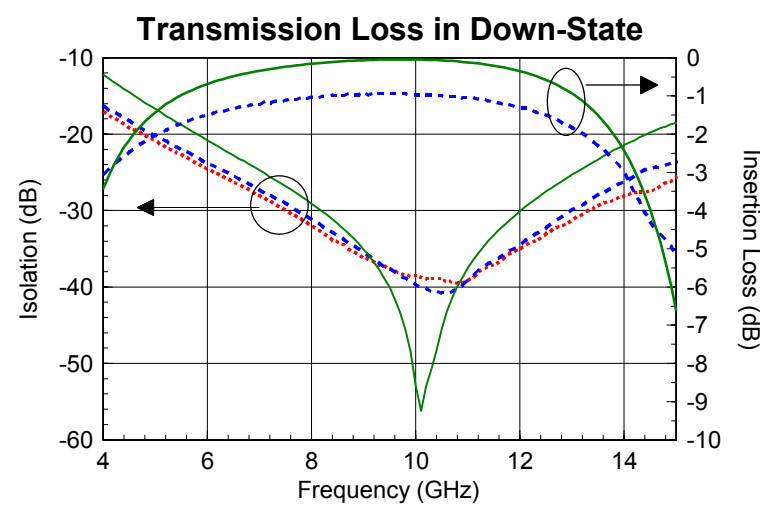

Fig. 7. Port 2 to port 1 (red dotted line) and port 2 to port 3 (blue dashed line) isolation are compared to simulated results with the switch in the down (actuated) state. Measured and simulated port 3 to port 1 insertion loss (blue dashed line) is also shown. Green solid lines are simulated data.

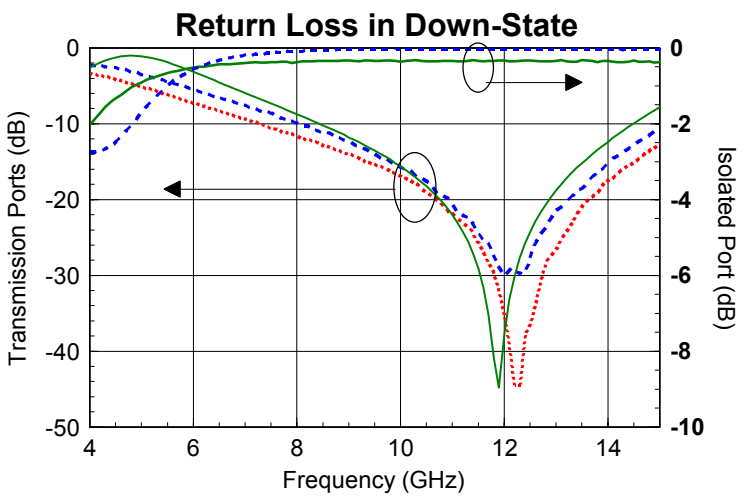

Fig. 8. Port 1 (red dotted line) and port 3 (blue dashed line) return losses are shown and compared to simulated results in the up-state. Return loss of the isolated Port 2 (top blue line) is also plotted. Green solid lines are simulated data. 


\section{CONCLUSION}

A SPDT RF MEMS switch, where an X-band signal can be switched between two output ports by applying a single bias voltage, was introduced. A capacitive MEMS shunt switch, which was used as a building block in the SPDT switch design, has also been measured and shown to have a low insertion loss of $0.25 \mathrm{~dB}$ in the up state with an isolation of $>30 \mathrm{~dB}$ in the down state. A $40 \mathrm{~V}$ bias was needed to actuate the switch. For the fabricated SPDT switch, the measured isolation of the isolated ports in the up state was $15 \mathrm{~dB}$ with an insertion loss of $1 \mathrm{~dB}$ through the transmitted port. In the down state, when a $35 \mathrm{~V}$ bias was applied, the isolation of the isolated ports was found to be $40 \mathrm{~dB}$. The insertion loss to the other port was again about $1 \mathrm{~dB}$.

As already stated, the advantage of this new SPDT switch design is that a signal can be routed between two ports using just a single bias. This can be attributed to the use of two capacitive bridge MEMS structures with identical geometries, one in series and one in shunt configuration off-set by a quarter-wavelength line section, in the design. It has been found though from the measured isolation plots, that the isolation is much higher in the down-state. This is to be expected since the parasitic coupling capacitance of the series beam to the center conductor underneath can be significant with gaps below $3 \mu \mathrm{m}$ when the switch is unactuated. A capacitive shunt switch inherently provides much better isolation at higher frequencies. This helps give the SPDT switch a much better isolation in the down-state at $10 \mathrm{GHz}$. Research will be continued to help improve the isolation in the up-state.

\section{ACKNOWLEDGEMENT}

This work was supported by the U.S. Army Space and Missile Defense Command. "Distribution A. Approved for Public Release; distribution unlimited."

\section{REFERENCES}

[1] K. Lee and B. Kim, "RF MEMS Switch for Wireless LAN Applications", Proceedings of the International Conference on MEMS, NANO and Smart Systems, pp. 100102, July 2003.

[2] M. Scardelleti, G. Ponchak, and N. Varaljay, "MEMS, KaBand Single-Pole Double-Throw (SPDT) Switch for Switched Line Phase Shifters", Antennas and Propagation Society International Symposium, Vol. 2, pp. 2-5, June 2002.

[3] J. Park, et al., "A 35-60 GHz Single -Pole Double-Throw (SPDT) Switching Circuit Using Direct Contact MEMS Switches and Double Resonance Technique", Transducers '03, Vol. 2, pp. 1796-1799, June 2003.

[4] D. Sievenpiper, et al., "MEMS-Based Switched Diversity Antenna at $2.3 \mathrm{GHz}$ for Automotive Applications", Wireless Personal Multimedia Communications, Vol. 2, pp. 762-765, Oct. 2003.

[5] B. Schauwecker, et al., "Single-Pole-Double-Throw Switch based on Toggle Switch", Electronics Letters, Vol. 39, No. 8, pp. 668 - 670, April 003.

[6] Advance Design Systems, version 2003A, Agilent Technologies, Palo Alto, CA 94304. 